\title{
Declinación en el uso de los métodos de revascularización y de angioplastia coronaria en Estados Unidos: Previsible pero muy preocupante
}

\author{
Annual decrease number of $\mathrm{PCl}$ procedures in USA: A Cause of Concern
}

Revista Argentina de Cardioangiología Intervencionista 2020;11(1):13-14. https://doi.org/10.30567/RAC1/202001/0013-0014

En el número de febrero de JAMA Cardiology $y^{1}$ se publicó una extensa base de datos de los cambios temporales de revascularización en EE.UU. en el período 2003-2016, cambios en revascularización que incluyen, como era de esperar, a las dos técnicas por todos conocidas: la cirugía de revascularización miocárdica (CABG) y la angioplastia coronaria (PCI).

Los autores dividen este período en 3 etapas: de 2003 a 2007, de 2008 a 2012 y de 2013 a 2016.

Excluyendo variaciones étnicas y raciales, que a nuestro país no le conciernen, es notable observar que la disminución se observa en ambos procedimientos de revascularización CABG y PCI, pero los cambios son más evidentes y pronunciados en la PCI.

En EE.UU., desde la introducción masiva de la PCI en la práctica clínica, se vio una disminución progresiva y constante de la CABG como método de revascularización.

Sin embargo, esto fue lo opuesto en el caso de la PCI, que evidenció un continuo crecimiento en las décadas $1980 / 1990 / 2000$.

Con la introducción de los stents liberadores de fármacos (DES), que disminuyeron significativamente la incidencia de nuevas revascularizaciones dentro del ler año, era de esperar un incremento aun mayor de los procedimientos de PCI. Esto, sin embargo, no parece ser así. La CABG registra decrecimiento entre el 2003 y el 2016, pero la curva es menos pronunciada que la del descenso de la PCI, como se puede observar en la figura central de dicho artículo.

En este período, con PCI hubo un aumento de procedimientos en pacientes con STEMI, infarto no STEMI, uso de FFR, soporte circulatorio y tratamiento de obstrucción total crónica, y una menor incidencia de procedimientos en pacientes estables, en el uso de stents metálicos desnudos (BMS) y tratamiento de vasos múltiples.

Con la CABG se observó un incremento en el tratamiento de infarto de miocardio, lesiones de 1 o 2 vasos, uso de conductos arteriales y tratamiento de shock cardiogénico. Ambas estrategias de revascularización presentaron aumento significativo de las comorbilidades.

La mortalidad hospitalaria observada mostró una disminución significativa con CABG y un incremento significativo con PCI que es difícil de explicar por los datos aportados en el manuscrito; sin embargo, el aumento de la mortalidad a 30 días con PCI se observó tanto en pacientes con IAM como en aquellos con angina inestable o estable.

La declinación de los procedimientos de revascularización miocárdica era esperable dado el resultado de los avances en el tratamiento médico de la enfermedad coronaria, sin embargo es indudable que esto afecta mucho más a la PCI que a la CABG dadas las características mucho más invasivas de esta última.

Además, hay que decir que esta observación abarca un período donde todavía no teníamos los resultados de varios estudios aleatorizados que, a mi criterio -siempre los editoriales expresan la opinión del autor-, fueron completamente negativos para PCI ya sea cuando se compararon con CABG o con tratamiento médico: SYNTAX, BEST, NOBLE, EXCEL, ORBITA e ISCHEMIA ${ }^{2-8}$.

Por ello es de esperar una declinación mayor en el uso de angioplastia en los años que vienen.

Creo que es necesario que todos los que estamos esta profesión y que hemos nacido con ella, médicos e industria fundamentalmente, repensemos qué hemos hecho en los últimos 15 años con la PCI para llegar a este punto. Es tiempo de reescribir la historia y pensar qué les vamos a dejar a los nuevos estudiantes de la carrera en los próximos años. Por el momento creo que, hasta que no sepamos qué nos pasó en estos últimos 15 años con la PCI y cuál va a ser el dispositivo más seguro y eficaz para utilizar en ella (DES y/o BMS), no deberíamos realizar ningún nuevo estudio aleatorizado comparativo entre nuestra especialidad y la CABG o el tratamiento médico.

Alfredo E. Rodríguez MD,PhD,FACC,FSCAI

Editor en Jefe Revista Argentina de Cardioangiología Intervencionista (RACI) 


\section{BIBLIOGRAFÍA}

1. Alkhouli J, Alqahtani F, Kalra A, et al. Trends in Characteristics and Outcomes of Patients Undergoing Coronary Revascularization in the United States, 2003-2016. JAMA Netw Open 2020 Feb 5;3(2):e1921326.

2. Mohr FW, Morice MC, Kappetein AP, et al. Coronary artery bypass graft surgery versus percutaneous coronary intervention in patients with three-vessel disease and left main coronary disease: 5-year follow-up of the randomised, clinical SYNTAX trial. Lancet 2013;381(9867):629-38.

3. Park SJ, Ahn JM, Kim YH, et al; BEST Trial Investigators. Trial of everolimus-eluting stents or bypass surgery for coronary disease. N Engl J Med 2015 Mar 26;372(13):1204-12.

4. Holm NR, Mäkikallio T, Lindsay MM, et al. Percutaneous coronary angioplasty versus coronary artery bypass grafting in the treatment of unprotected left main stenosis: updated 5-year outcomes from the randomised, non-inferiority NOBLE trial. Lancet 2020;395(10219):191-9.

5. Stone GW, Kappetein AP, Sabik JF, et al. Five-Year Outcomes after PCI or CABG for Left Main Coronary Disease. N Engl J Med 2019;381(19):1820-30.

6. Al-Lamee R, Thompson D, Dehbi H, et al Percutaneous coronary intervention in stable angina (ORBITA): a double-blind, randomised controlled trial. Lancet 2018, January 6, 391;31-41.

7. Rodríguez AE. The ORBITA trial: Why is it not the last nail for coronary angioplasty in stable angina patients? Cardiovasc Revasc Med 2019 Jan;20(1):80-1.

8. Presented by Judith S. Hochman at the American Heart Association Annual Scientific Sessions (AHA 2019), Philadelphia, PA, November 16, 2019. International Study of Comparative Health Effectiveness with Medical and Invasive Approaches - ISCHEMIA. 